\title{
Renal-protective effect of thalidomide in streptozotocin-induced diabetic rats through anti-inflammatory pathway
}

This article was published in the following Dove Press journal:

Drug Design, Development and Therapy

\author{
Hongxia Zhang' \\ Yanlan Yang ${ }^{2}$ \\ Yanqin Wang' \\ Baodong Wang' \\ Rongshan $\mathrm{Li}^{\prime}$ \\ 'Department of Nephrology, \\ ${ }^{2}$ Department of Endocrinology, \\ Affiliated People's Hospital of Shanxi \\ Medical University, Shanxi Provincial \\ People's Hospital, Taiyuan, Shanxi, \\ People's Republic of China
}

Correspondence: Rongshan Li Department of Nephrology, Affiliated People's Hospital of Shanxi Medical University, Shanxi Provincial People's Hospital, No 29, Shuangta Road, Taiyuan, Shanxi 0300I2, People's Republic of China

Tel +86 I8734195439

Email rongshanlil3@I63.com
Background: Diabetic nephropathy (DN) is a major microvascular complication in diabetes. An increasing body of evidence has shown that DN is related to chronic inflammation, kidney hypertrophy, and fibrosis. While thalidomide has been shown to have anti-inflammatory and antifibrotic effects, the effects of thalidomide on the pathogenesis of DN are unclear. This study was undertaken to explore whether thalidomide has renal-protective effects in diabetic rats.

Methods: Male Sprague Dawley rats were injected intraperitoneally with $50 \mathrm{mg} / \mathrm{kg}$ streptozotocin to induce diabetes. Diabetic rats were treated with thalidomide $(200 \mathrm{mg} / \mathrm{kg} / \mathrm{d})$ for 8 weeks, and then blood and urine were collected for measurement of renal function-related parameters. Histopathology, immunohistochemistry, enzyme-linked immunosorbent assay, and Western blot analyses were performed to assess renal proinflammatory cytokines, fibrotic protein, and related signaling pathways.

Results: Diabetic rats exhibited obvious renal structural and functional abnormalities, as well as renal inflammation and fibrosis. Compared with diabetic control rats, those treated with thalidomide showed significantly improved histological alterations and biomarkers of renal function, as well as reduced expression of renal inflammatory cytokines, including NF- $\kappa \mathrm{B}$ and MCP-1. Furthermore, renal fibrotic proteins, such as TGF- $\beta 1$, T $\beta$ RII, T $\beta$ RI, smad3, collagen IV, and fibronectin were also remarkably suppressed. Treatment with thalidomide markedly stimulated the phosphorylation of AMPK $\alpha$.

Conclusion: In this study, thalidomide suppressed the inflammatory and fibrotic processes in $\mathrm{DN}$. These effects were partly mediated by the activation of AMPK $\alpha$, and inhibition of the NF- $\mathrm{KB}$ / MCP- 1 and TGF- $\beta 1 /$ Smad signaling pathways. These results suggest that thalidomide may have therapeutic potential in diabetic renal injury through the anti-inflammatory pathway.

Keywords: diabetic nephropathy, thalidomide, AMPK, NF- $\kappa B$, TGF- $\beta 1$

\section{Introduction}

Diabetic nephropathy (DN) is a lethal complication of diabetes and a leading cause of end-stage renal disease worldwide. ${ }^{1-3}$ This progressive kidney disease is mainly caused by alterations in the glomerular capillary and tubular structure and function induced by disturbed glucose homeostasis. ${ }^{4}$ A typical morphological change in DN is abnormal extracellular matrix (ECM) accumulation, which contributes to basement membrane thickening and consequent glomerulosclerosis and tubulointerstitial fibrosis. ${ }^{5,6}$

Although major advances in the diagnosis and treatment of DN have been made over the past few decades, it is still very difficult to significantly reduce mortality in this population. ${ }^{7,8}$ The pathogenesis of DN remains unclear. Several studies have 
demonstrated that chronic inflammation, oxidative stress, fibrosis, and dyslipidemia induced by hyperglycemia lead to renal injury through different signaling pathways, which may be pivotal factors in the occurrence and development of DN. ${ }^{9,10}$

Recently, thalidomide has been shown to have antiinflammatory and antifibrotic properties in animal models of myocardial, peritoneal, and pulmonary fibrosis. ${ }^{11-15}$ It also protects against tubulointerstitial injury in adenine-induced chronic kidney disease in mice. ${ }^{16}$ Accordingly, previous studies have demonstrated that thalidomide has antiinflammatory and antifibrotic effects in diabetic retinopathy, neuropathy, and cardiomyopathy by suppressing the levels of proinflammatory and profibrotic cytokines such as TNF- $\alpha$, IL-1b, IL-6, and TGF- $\beta 1 .{ }^{17-19}$ However, its effects on DN remain unknown. The purpose of this study was to investigate whether thalidomide prevents the development of renal injury in streptozotocin (STZ)-induced diabetic rats.

\section{Methods}

\section{Animals}

Healthy 8- to 10-week-old male, specific pathogen-free Sprague Dawley (SD) rats (weighing 200-230 g) were obtained from the Experimental Animal Center of Shanxi Medical University (Taiyuan, People's Republic of China). All experiments were performed according to protocols outlined by the Institutional Animal Care and Use Committee of Shanxi Medical University and were approved by the Ethics Committee of Shanxi Medical University. The animals were housed at a room temperature of $22^{\circ} \mathrm{C} \pm 2{ }^{\circ} \mathrm{C}$ and $45 \% \pm 5 \%$ humidity, and fed a normal diet during the experiment.

\section{Experimental protocol}

Rats $(n=18)$ were rendered diabetic by a single intraperitoneal injection of STZ (Sigma-Aldrich, Inc., St Louis, MO, USA) diluted in citrate buffer $(10 \mathrm{mM}, \mathrm{pH} 4.5)$ at a dose of $50 \mathrm{mg} / \mathrm{kg}$. The normal control group $(\mathrm{NC}, \mathrm{n}=18)$ was injected with an equal volume of citrate buffer. At 72 hours after STZ injection, blood glucose was measured after 8 hours of food deprivation and diabetes was confirmed if glyce$\mathrm{mia} \geq 16.7 \mathrm{mmol} / \mathrm{L}$. Four weeks after STZ injection, diabetic rats were randomly divided into two groups (originally, $n=9$ each). The diabetic control+thalidomide group (DC+Thd) was treated with an oral dose of thalidomide $(200 \mathrm{mg} / \mathrm{kg} / \mathrm{d}$, Changzhou Qianhong Biopharma Company, Changzhou, People's Republic of China) dissolved in corn oil for 8 weeks. The DC group was treated with an equal volume of corn oil without thalidomide. $\mathrm{NC}(\mathrm{n}=9)$ rats were also treated with thalidomide ( $\mathrm{NC}+\mathrm{Thd}, \mathrm{n}=9$ ) according to the same experimental protocol as for the diabetic rats. The weight and blood glucose of all animals were evaluated once a week. The dosage of thalidomide was based on previous studies on diabetic animals. ${ }^{20}$

At day 56 of drug administration, animals were housed in individual metabolic cages and 24-hour urine samples were collected. Arterial blood from the abdominal aorta was collected before the animals were sacrificed. The left kidneys were rinsed with cold isotonic saline and immediately frozen in liquid nitrogen until analysis. The right kidneys were weighed and fixed in 10\% neutral-buffered formalin.

\section{Biochemical estimations in plasma and urine samples}

The blood samples were centrifuged at 3,000 g for 10 minutes. Plasma glucose, blood urea nitrogen (BUN), and serum creatinine ( $\mathrm{SCr}$ ) were measured by an automatic biochemical analyzer (Hitachi 917, Tokyo, Japan). Serum insulin was determined using "Mercodia Rat Insulin ELISA Kit" (Mercodia, Uppsala, Sweden). Urine albumin was measured using an ELISA kit from CUSABIO Engineering Co. (Wuhan, People's Republic of China).

\section{Histopathological analysis}

For histological analysis, right kidneys were fixed in 10\% neutral-buffered formalin, embedded in paraffin, and cut into $3 \mu \mathrm{m}$ sections. The sections were stained with hematoxylin and eosin (H\&E), periodic acid-Schiff (PAS), and Masson, respectively. Glomerulosclerosis was defined as the percentage of ECM deposition and mesangial expansion and evaluated for 20 cortical fields: 0, normal glomeruli; 1 , matrix expansion occurring in up to $50 \%$ of the glomerulus; 2 , matrix expansion occurring in $50 \%-75 \%$ of the glomerulus; 3, matrix expansion occurring in $75 \%-100 \%$ of the glomerulus. ${ }^{21}$ The relative mesangial area was expressed as mesangial area/glomerular area (\%). The degree of tubulointerstitial damage including tubular dilation, tubular atrophy, cast formation, and interstitial mononuclear cell and ECM accumulation (interstitial volume) was scored $(1=<10 \%$; $2=10 \%-25 \% ; 3=26 \%-50 \%, 4=51 \%-75 \%, 5=76 \%-95 \%$, and $6=>95 \%) .{ }^{22}$ All images were assessed using Image Pro Plus 6.1 (Media Cybernetics LP, MD, USA). Quantitative analysis was conducted in a blinded manner.

\section{Immunohistochemical analysis}

After deparaffinization and hydration, the slides were washed in Tris-buffered saline (TBS). Endogenous peroxidase activity was quenched by incubating the slides in methanol solution containing $3 \% \mathrm{H}_{2} \mathrm{O}_{2}$. After an overnight incubation 
with primary antibodies (TGF- $\beta 1$, catalog no ab92486, 1:500, Abcam, Eugene, OR, USA; MCP-1, catalog no ab25124, 1:500, Abcam; NF-кB, catalog no ab16502, 1:500, Abcam; fibronectin, catalog no 15613-1-AP, 1:300, Proteintech, Wuhan, People's Republic of China; collagen IV, catalog no 19797-1-AP, 1:300, Proteintech) at $4^{\circ} \mathrm{C}$, the slides were washed in TBS. The horseradish peroxidase-conjugated secondary antibody was added and reacted at room temperature for 1 hour. The slides were then washed in TBS and incubated with diaminobenzidine tetrahydrochloride as the substrate, and finally counterstained with hematoxylin. Negative control slides without primary antibodies were made in the same manner. Twenty glomeruli were selected randomly from each group and positive staining within each glomerulus was measured by Image Pro Plus 6.1. Quantitative analysis was conducted in a blinded manner.

\section{Western blot analysis}

The left kidneys were extracted with RIPA lysis buffer (Beyotime Biotech, Jiangsu, People's Republic of China) and centrifuged at $12,000 \mathrm{~g}$ for 45 minutes at $4{ }^{\circ} \mathrm{C}$ to obtain the supernatant. The protein concentration was determined using a BCA Protein Assay Kit (Beyotime Biotech). Samples containing $50 \mu \mathrm{g}$ of protein were resolved by $12 \%$ SDS-PAGE electrophoresis and transferred to nitrocellulose membranes (Whatman International Ltd., Maidstone, Germany). After blocking for 1 hour, the membranes were incubated with the following primary antibodies at $4^{\circ} \mathrm{C}$ overnight: TGF- $\beta 1$ (catalog no ab92486, 1:1,000, Abcam); Smad3 (catalog no ab28379, 1:1,000, Abcam); phospho (p)-Smad3 (catalog no ab63403, 1:1,000, Abcam); T $\beta$ RI (catalog no sc-101574, 1:200, Santa Cruz Biotechnology); T $\beta$ RII (catalog no sc-17791, 1:200, Santa Cruz Biotechnology); NF-кB p65 (catalog no \#4764, 1:1,000, Cell Signaling Technology, Danvers, MA, USA); AMPK $\alpha$ (catalog no \#2532, 1:1,000, Cell Signaling Technology); phospho (p)-AMPK $\alpha$ (Thr172) (catalog no \#50081, 1:1,000, Cell Signaling Technology); $\beta$-actin (as an internal loading control, catalog no ab8226, 1:1,000, Abcam); GAPDH (as an internal loading control, catalog no \#5174, 1:1,000, Cell Signaling Technology). After being washed in TBST, the membranes were incubated with corresponding horseradish peroxidase-conjugated secondary antibodies at room temperature for 1.5 hours and analyzed by the Quantity One analysis system (Bio-Rad, Hercules, CA, USA).

\section{ELISA}

The level of activated AMPK $\alpha$ in renal cortex tissue was determined by a sandwich ELISA to measure p-AMPK $\alpha$ (Jianglai Bioscience Co., Shanghai, People's Republic of China) according to the manufacturer's protocol.

\section{Statistical analysis}

Statistical analysis was performed using SPSS 19.0 software (IBM, Armonk, NY, USA). The values are presented as the mean \pm standard error of the mean. Differences between the groups were measured by one-way analysis of variance and post hoc analysis with Bonferroni correction. Data were considered statistically significant at $P<0.05$.

\section{Results \\ Physical and biochemical characteristics of diabetic rats}

During the experiment, two rats in the DC group and one in the DC+Thd group died. Kidneys in the DC group were heavier than those in the NC group. Right kidney weight, ratio of kidney weight to body weight $(\mathrm{K} / \mathrm{W})$, plasma glucose, $\mathrm{SCr}$, BUN, urine albumin, and 24-hour urine volume were significantly higher for the DC group than for the NC group $(P<0.01)$ (Table 1). Meanwhile, body weight and serum insulin were markedly decreased in the DC group compared with the NC group $(P<0.01)$ (Table 1).

Table I Changes in renal function and biochemical parameters in each group

\begin{tabular}{|c|c|c|c|c|}
\hline Characteristic & $N C(n=9)$ & NC+Thd $(n=9)$ & $D C(n=7)$ & DC + Thd $(n=8)$ \\
\hline Body weight (g) & $361.14 \pm 4.42$ & $362.83 \pm 4.90$ & $297.29 \pm 9.44^{*}$ & $301.50 \pm 8.15$ \\
\hline Right kidney weight (g) & $1.32 \pm 0.04$ & $1.34 \pm 0.06$ & $1.75 \pm 0.07 *$ & $1.54 \pm 0.10$ \\
\hline $\mathrm{K} / \mathrm{W}(\mathrm{mg} / \mathrm{g})$ & $3.65 \pm 0.08$ & $3.65 \pm 0.13$ & $5.91 \pm 0.21 *$ & $4.97 \pm 0.25 * *$ \\
\hline BUN (mmol/L) & $5.98 \pm 0.19$ & $5.87 \pm 0.20$ & $9.01 \pm 0.35 *$ & $7.85 \pm 0.3 \mid * * *$ \\
\hline $\mathrm{SCr}(\mu \mathrm{mol} / \mathrm{L})$ & $30.68 \pm 1.27$ & $29.74 \pm 1.75$ & $53.46 \pm 2.21^{*}$ & $40.97 \pm 2.03 * *$ \\
\hline Serum insulin $(\mu g / L)$ & $2.34 \pm 0.07$ & $2.31 \pm 0.08$ & $0.99 \pm 0.06 *$ & $1.02 \pm 0.05$ \\
\hline Plasma glucose (mmol/L) & $4.99 \pm 0.11$ & $5.05 \pm 0.17$ & $20.90 \pm 1.07^{*}$ & $19.76 \pm 1.06$ \\
\hline Urine albumin (mg/24 h) & $4.84 \pm 0.18$ & $4.93 \pm 0.23$ & $31.52 \pm 2.19 *$ & $21.36 \pm 1.95 * *$ \\
\hline $24-\mathrm{h}$ urine volume $(\mathrm{mL} / \mathrm{d})$ & $8.47 \pm 0.46$ & $8.60 \pm 0.43$ & $87.19 \pm 2.6 I^{*}$ & $83.95 \pm 4.26$ \\
\hline
\end{tabular}

Notes: Data are expressed as mean \pm SEM ( $n=7-9)$. $* p<0.01$ versus NC group; $* * P<0.01$ versus DC group; $* * * p<0.05$ versus $D C$ group.

Abbreviations: BUN, blood urea nitrogen; DC, diabetic control; K/W, kidney weight/body weight ratio; NC, normal control; SCr, serum creatinine; SEM, standard error of the mean; Thd, thalidomide. 


\section{Thalidomide treatment improved renal function in diabetic rats}

After 8 weeks of thalidomide treatment, $\mathrm{SCr}, \mathrm{K} / \mathrm{W}$, and albuminuria were significantly decreased in the $\mathrm{DC}+\mathrm{Thd}$ group compared with those in the DC group $(P<0.01)$ (Table 1). There were no differences in blood glucose, serum insulin, body weight, or kidney weight between the two groups $(P>0.05)$ (Table 1). Despite the same degree of hyperglycemia in the DC and DC+Thd groups, thalidomide treatment significantly reduced $\mathrm{SCr}$ and albuminuria.

\section{Thalidomide treatment reduced the renal histological alterations in diabetic rats}

There were no differences in the histology characteristics between the $\mathrm{NC}$ and $\mathrm{NC}+\mathrm{Thd}$ groups. In contrast, mesangial matrix expansion and basement membrane thickening in glomeruli and tubules were greater in the DC group than in the NC group. After treatment with thalidomide, renal morphological alterations were considerably improved in the DC+Thd group, as indicated by H\&E, PAS, and Masson staining, respectively (Figure 1A). Notably, glomerular mesangial matrix expansion $(P<0.01)$ and collagen deposition $(P<0.05)$ were substantially reduced in the DC+Thd group compared with the DC group (Figure 1B). These results confirmed that thalidomide reduced diabetic renal damage.

\section{Effects of thalidomide on renal inflammatory and fibrotic cytokine expression}

To investigate the effects of thalidomide on renal inflammation and fibrosis, the expression levels of TGF- $\beta 1, N F-\kappa B$, $\mathrm{MCP}-1$, collagen IV, and fibronectin in diabetic kidneys were determined by immunohistochemistry (Figures 2A and 3A). Consistent with the changes in the diabetes-induced histological alterations, the levels of the profibrotic growth factor TGF- $\beta 1$ and associated ECM collagen IV, fibronectin, and
A
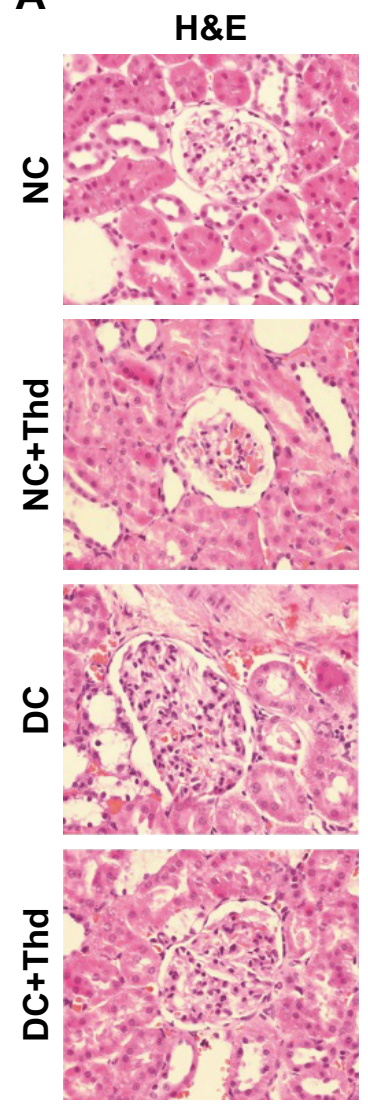

PAS
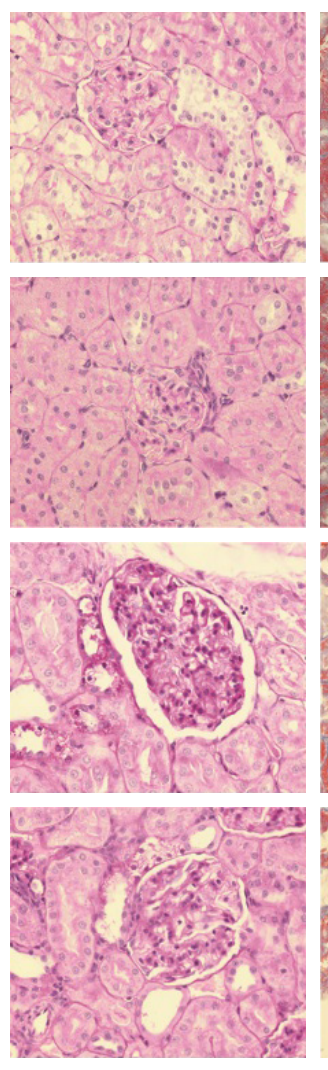

Masson
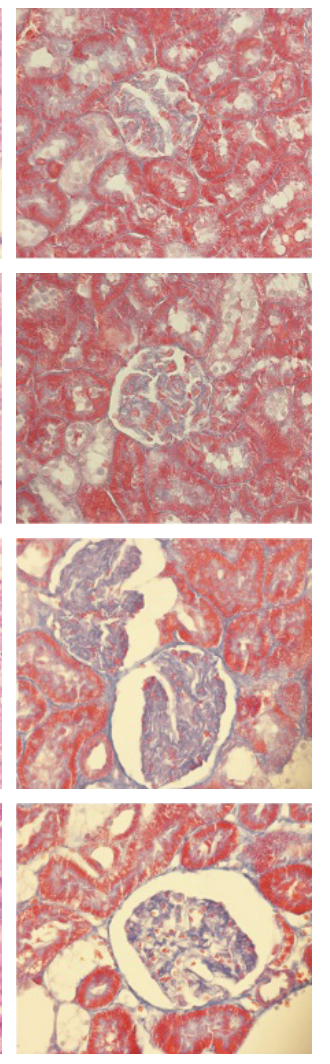

B
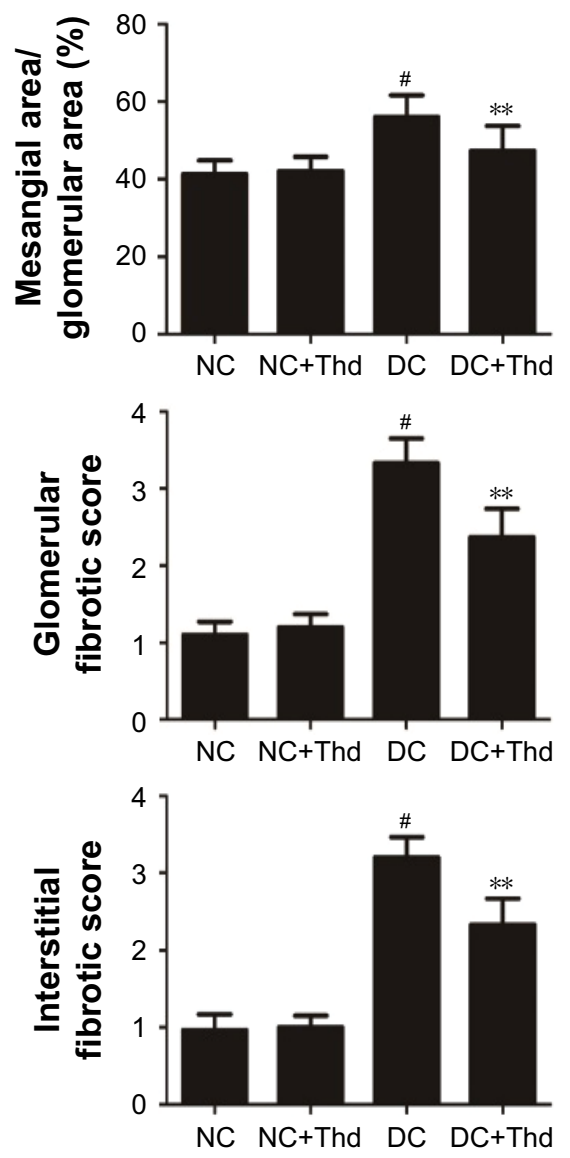

Figure I Renal histology after treatment with or without thalidomide.

Notes: (A) Representative micrographs of H\&E, PAS, and Masson staining. Original magnification: 400x. (B) Quantitative analyses of mesangial area and Masson staining of glomerular and tubulointerstitial lesions. Values are expressed as mean $\pm S E M(n=5)$. ${ }^{*} P<0.0$ I versus NC group; $* * P<0.01$ versus $D C$ group.

Abbreviations: DC, diabetic control; H\&E, hematoxylin and eosin; NC, normal control; PAS, periodic acid-Schiff; SEM, standard error of the mean; Thd, thalidomide. 
A

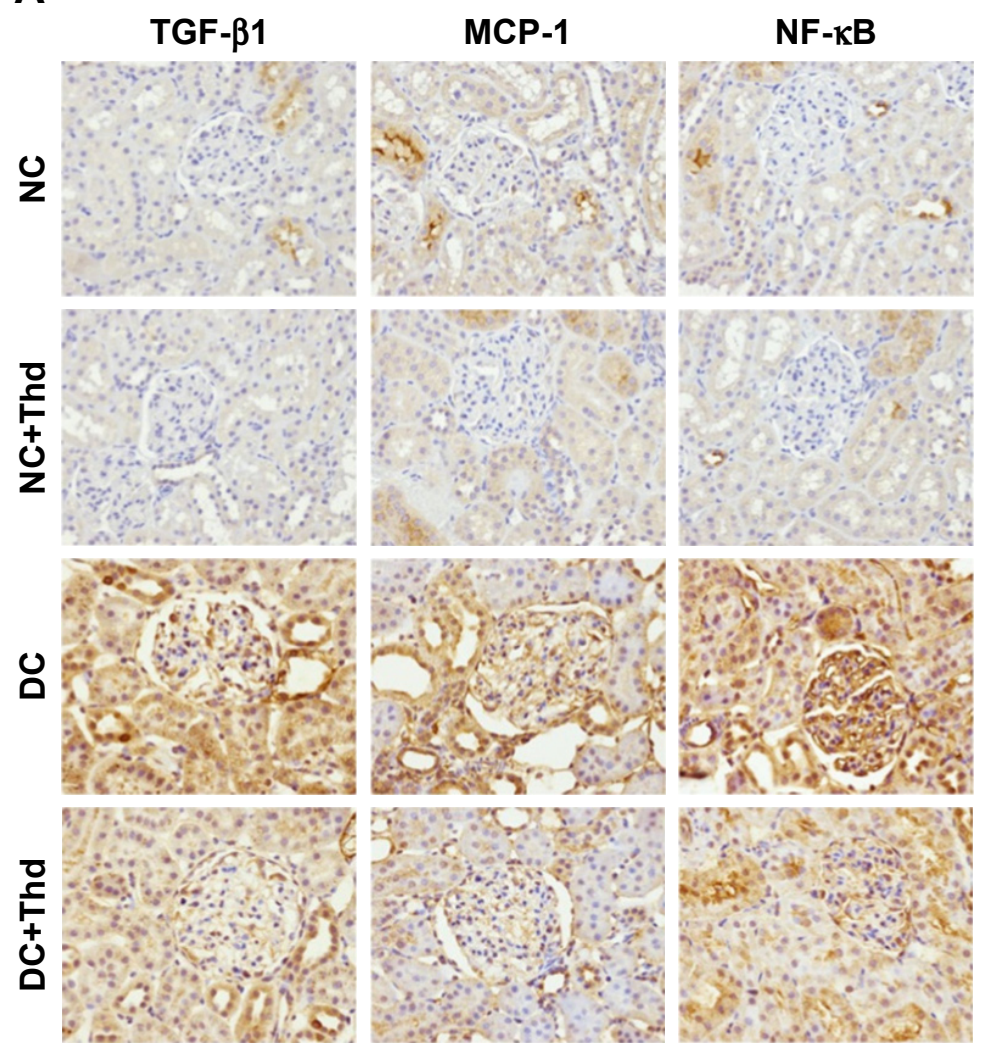

B
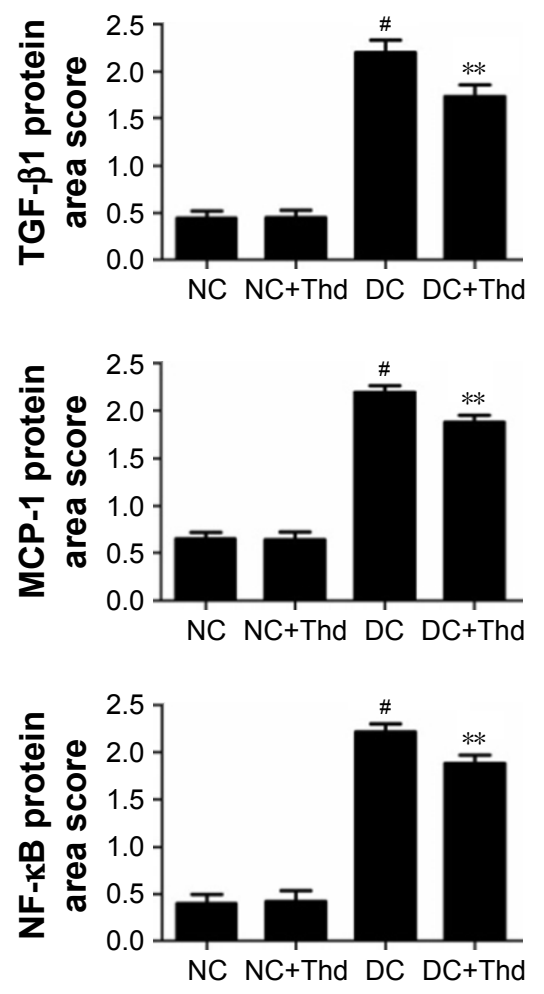

Figure 2 Expression of TGF- $\beta$ I, MCP-I, and NF- $\mathrm{KB}$ in kidneys.

Notes: (A) Immunohistochemistry of TGF- $\beta$ I, MCP-I, and NF- $\kappa B$. Original magnification 400x. (B) TGF- $\beta$ I, MCP-I, and NF- $\kappa B$ expression were semiquantitatively analyzed. Values are expressed as mean \pm SEM $(n=5)$. ${ }^{*}<0.01$ versus $N C$ group; $* * P<0.01$ versus $D C$ group.

Abbreviations: DC, diabetic control; NC, normal control; SEM, standard error of the mean; Thd, thalidomide.

inflammatory factors $\mathrm{NF}-\kappa \mathrm{B}$ and $\mathrm{MCP}-1$ in the DC group were markedly higher than those in the $\mathrm{NC}$ and $\mathrm{NC}+\mathrm{Thd}$ groups $(P<0.01)$. After thalidomide treatment, the expression levels of all of the diabetes-induced renal fibrotic and inflammatory cytokines were significantly decreased $(P<0.05)$ (Figures $2 \mathrm{~B}$ and $3 \mathrm{~B})$. These results suggest that thalidomide has anti-inflammatory and antifibrotic effects on diabetic renal injury.

\section{Thalidomide treatment activated AMPK and suppressed the TGF- $\beta$ I/Smad signaling pathway in diabetic rats}

The TGF- $\beta 1 /$ Smad signaling pathway plays a vital role in the pathogenesis of renal fibrosis. Compared with the values in the NC and NC+Thd groups, TGF- $\beta 1, \mathrm{~T} \beta \mathrm{RI}, \mathrm{T} \beta \mathrm{RII}$, and p-Smad3 expression in the DC group were significantly increased. After treatment with thalidomide, the expression levels of TGF- $\beta 1, T \beta R I$, T $\beta$ RII, and $p$-Smad 3 were markedly decreased $(P<0.05)$ (Figures $4 \mathrm{~A}$ and $\mathrm{B}$ and $5 \mathrm{~A}$ and $\mathrm{B}$ ). The expression of the inflammatory cytokine NF- $\kappa B$ showed the same trend $(P<0.01)$ (Figure 6A and $\mathrm{B})$. The expression of $\mathrm{p}$-AMPK $\alpha$ was significantly decreased in the DC group compared with that in the $\mathrm{NC}$ and $\mathrm{NC}+\mathrm{Thd}$ groups as demonstrated by both Western blot and ELISA $(P<0.01)$. Treatment with thalidomide remarkably upregulated the expression of p-AMPK $\alpha(P<0.05)$ (Figure 6C, D and E). These results showed that the inhibition of renal inflammation and fibrosis by thalidomide may be attributable to the suppression of TGF- $\beta 1 /$ Smad and the activation of AMPK signaling pathways.

\section{Discussion}

This is the first report to identify the beneficial effects of thalidomide against renal injury in diabetic rats. After diabetic rats were treated with thalidomide for 8 weeks, biochemical markers of renal function and renal morphological abnormalities were effectively improved. The present results suggest that thalidomide may exert these protective effects by upregulating $\mathrm{p}-\mathrm{AMPK} \alpha$ and inhibiting NF- $\mathrm{KB} / \mathrm{MCP}-1$ and TGF- $\beta 1 / \mathrm{Smad} 3$ signaling pathways.

$\beta$ cell destruction and insulin secretory deficiency are the main pathological characteristics of STZ-induced diabetic 

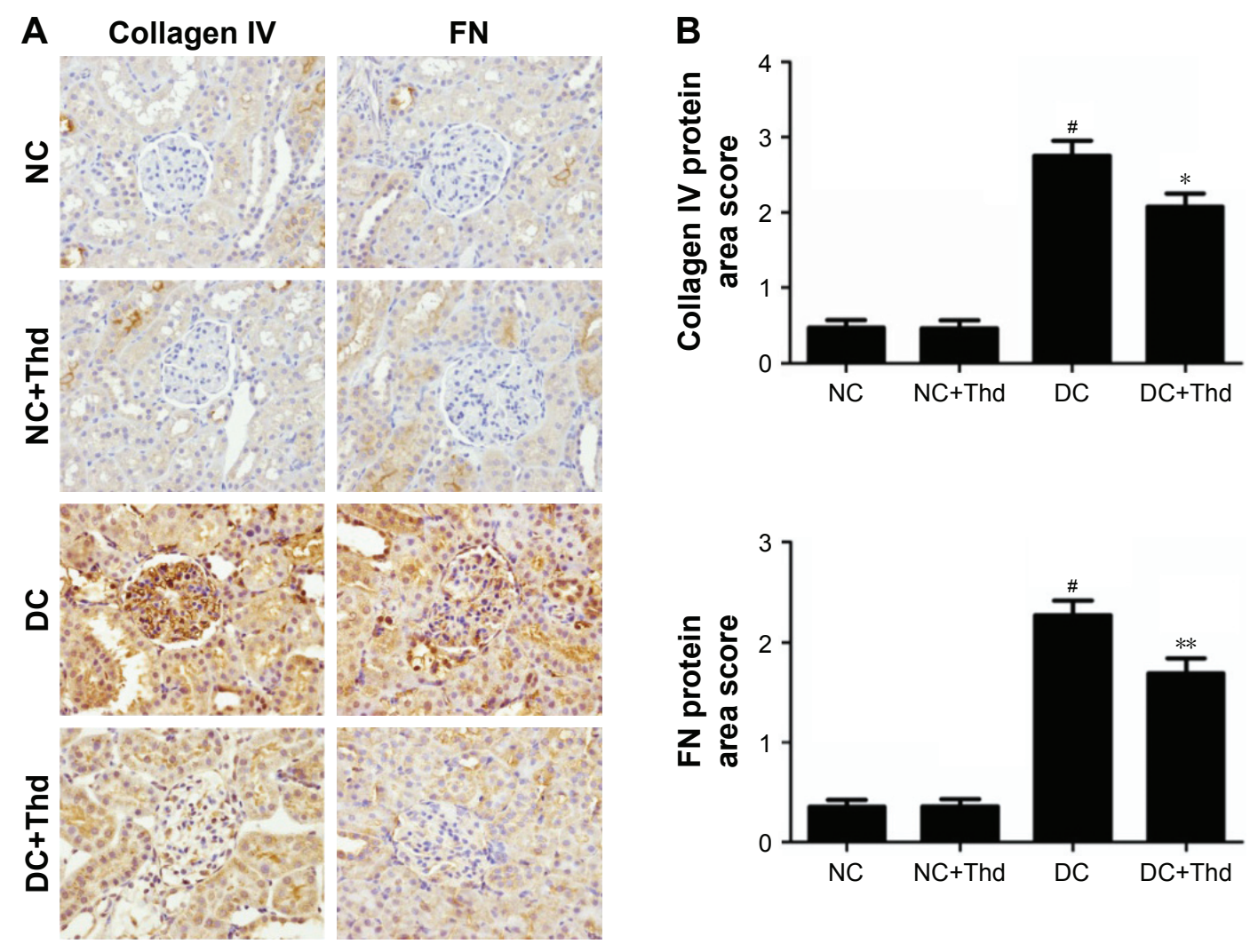

Figure 3 Expression of collagen IV and fibronectin in kidneys.

Notes: (A) Immunohistochemistry of collagen IV and fibronectin. Original magnification 400x. (B) Collagen IV and fibronectin expression were semiquantitatively analyzed. Values are expressed as mean \pm SEM $(n=5)$. ${ }^{P}<<0.0$ I versus NC group; $* P<0.05$ versus $D C$ group; $* * P<0.0$ I versus $D C$ group.

Abbreviations: DC, diabetic control; NC, normal control; SEM, standard error of the mean; Thd, thalidomide.

A

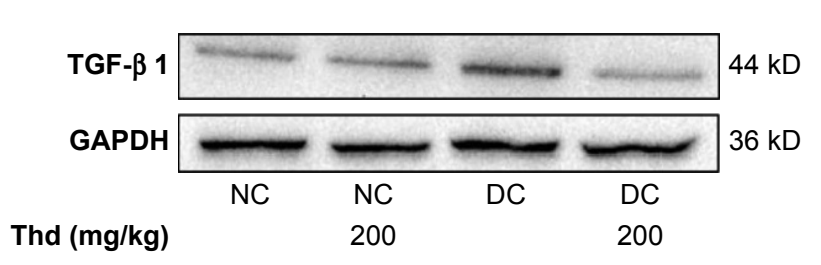

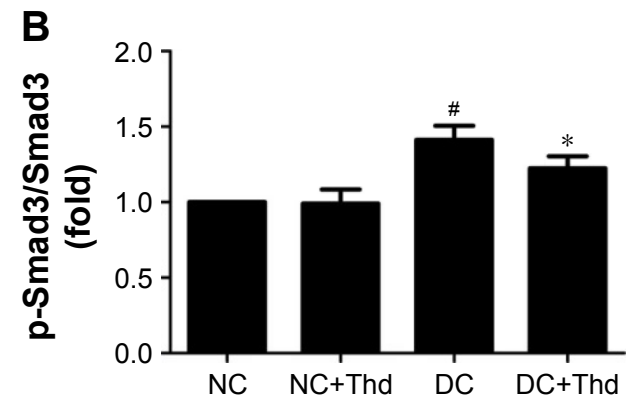

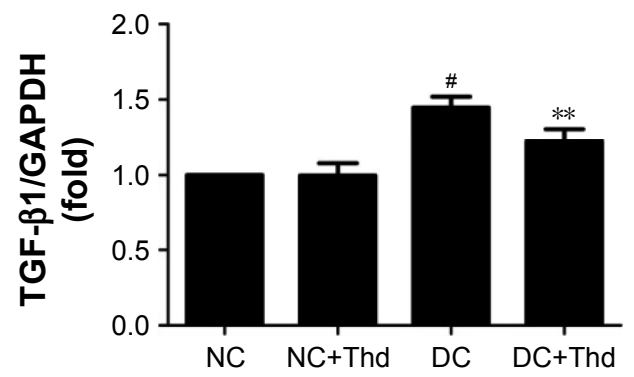

Figure 4 Effects of thalidomide on the expression of TGF- $\beta$ I, Smad3, and p-Smad3 by Western blot analysis.

Notes: (A) TGF- $\beta$ I, Smad3, and p-Smad3 expression in kidneys. (B) Band density analysis of TGF- $\beta$ I, Smad3, and $p-S m a d 3$. Values are expressed as mean \pm SEM ( $\mathrm{n}=5$ ). ${ }^{*} P<0.0$ I versus $\mathrm{NC}$ group; $* P<0.05$ versus $D C$ group; $* * P<0.01$ versus $D C$ group.

Abbreviations: DC, diabetic control; NC, normal control; p-Smad3, phospho-Smad3; SEM, standard error of the mean; TGF- $\beta$ I, transforming growth factor $\beta$ I; Thd, thalidomide. 
A

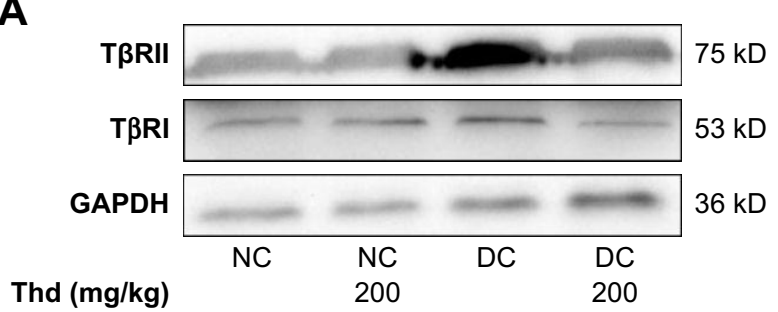

B
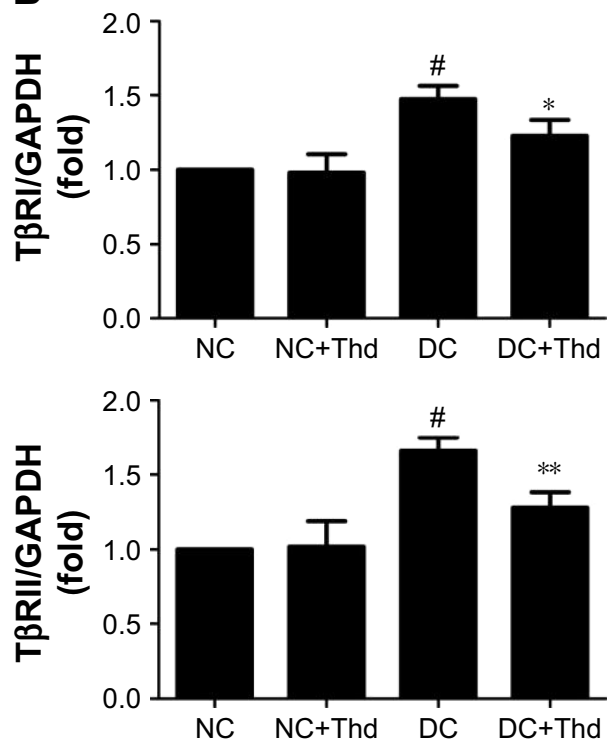

Figure 5 Effects of thalidomide on the expression of T $\beta R I I$ and T $\beta R I$ by Western blot analysis.

Notes: (A) T $\beta R I$ and T $\beta R I$ expression in kidneys. (B) Band density analysis of T $\beta R I I$ and T $\beta R I$. Values are expressed as mean $\pm S E M$ ( $n=5$ ). ${ }^{\#}<0.0 \mathrm{I}$ versus NC group; $* P<0.05$ versus $D C$ group; $* * P<0.0$ I versus $D C$ group.

Abbreviations: DC, diabetic control; NC, normal control; SEM, standard error of the mean; T $\beta$ RII, transforming growth factor- $\beta$ receptor II; T $\beta$ RI, transforming growth factor- $\beta$ receptor I; Thd, thalidomide.

A

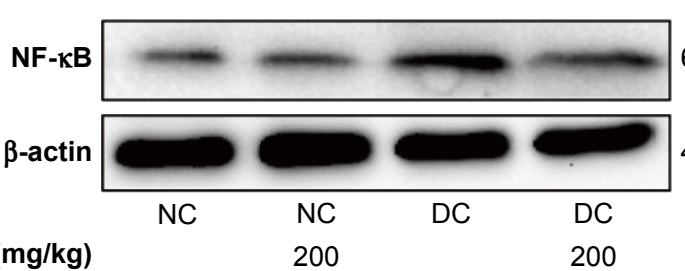

C

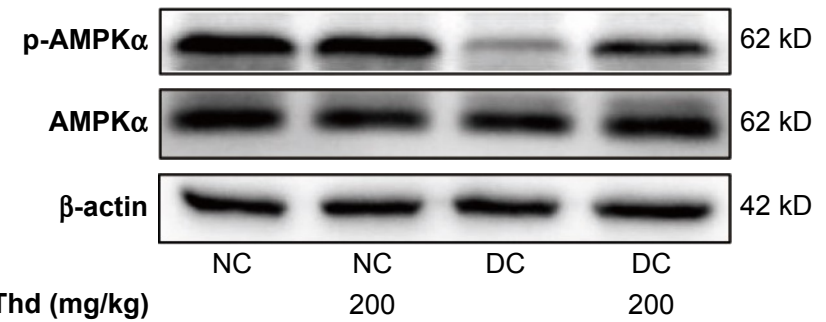

B
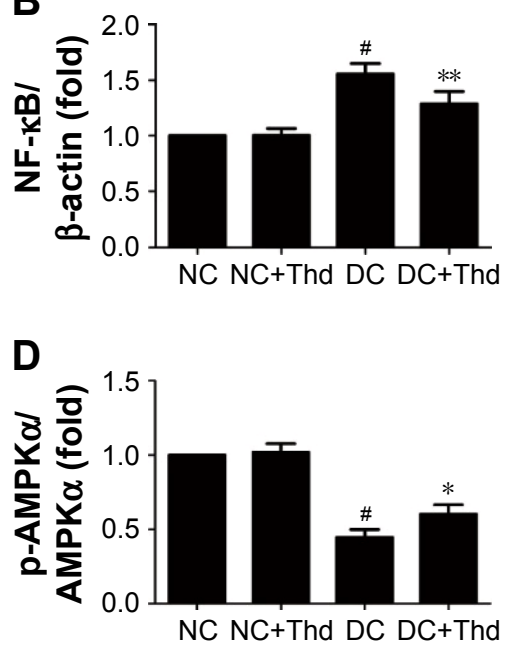

$\mathbf{E}$

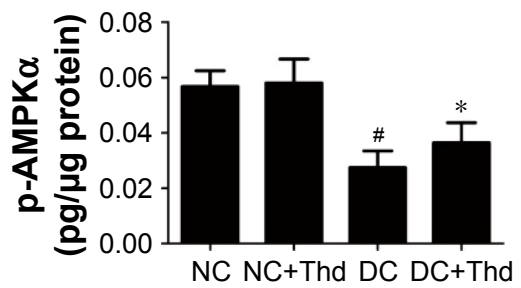

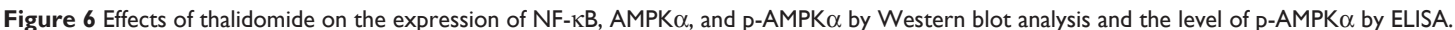

Notes: (A) NF-KB expression in kidneys. (B) Band density analysis of NF- $K B$. (C) AMPK $\alpha$ and $\mathrm{p}-\mathrm{AMPK} \alpha$ expression in kidneys. (D) Band density analysis of p-AMPK $\alpha /$ AMPK $\alpha$. (E) $P$-AMPK $\alpha$ expression in kidneys by ELISA. Values are expressed as mean $\pm S E M(n=4)$. ${ }^{*}<0.0$ I versus $N C$ group; $* P<0.05$ versus $D C$ group; $* * P<0.0$ I versus DC group.

Abbreviations: AMPK $\alpha$, AMP-activated protein kinase $\alpha$; DC, diabetic control; NC, normal control; NF- $\mathrm{B}$, nuclear factor- $\mathrm{B}$; $\mathrm{p}-\mathrm{AMPK} \alpha$, phospho-AMPK $\alpha$; SEM, standard error of the mean; Thd, thalidomide. 
rats. $^{23,24}$ These rats develop symptoms similar to those in human diabetes, such as polydipsia, polyuria, hyperglycemia, and body weight loss. Meanwhile, renal injury develops under these conditions. In the present study, plasma glucose, BUN, SCr, K/W ratio, albuminuria, and 24-hour urine volume were significantly increased in diabetic rats. Accordingly, renal inflammation and structural abnormalities and fibrosis were greater than those in normal rats. These results suggest that this was a good model of DN. Notably, to avoid intrinsic nephrotoxic effects, we acquired data 4 weeks after STZ injection when the kidneys have recovered from the acute renal injury caused by STZ. ${ }^{24-26}$ Thus, we assume that the renal damage in our model was solely due to the diabetic status.

In the present study, after diabetic rats were treated with thalidomide for 8 weeks, renal function parameters were markedly improved. Diabetic rats showed significant reductions in SCr, BUN, and albuminuria. Further, thalidomide treatment reduced renal inflammatory cytokines and histological injuries. However, thalidomide treatment did not reduce plasma glucose or body weight compared with those in the DC rats. These results suggest that the protective effect of thalidomide on $\mathrm{DN}$ is independent of glycemic regulation and body weight loss.

Chronic inflammation plays a pivotal role in the pathogenesis of DN. Various inflammatory molecules, such as adhesion molecules and proinflammatory cytokines, have been demonstrated to play significant roles in the setting of DN. ${ }^{9,10} \mathrm{NF}-\kappa \mathrm{B}$, which has been recognized as a central signaling pathway in inflammation, is usually activated by a wide variety of stress stimuli, including diabetes, obesity, and oxidative stress. Activation of NF- $\kappa B$ can induce the transcription of many cytokines such as MCP-1, TNF- $\alpha$, and the interleukin system. ${ }^{27}$ Meanwhile, macrophage recruitment to the kidneys can be accelerated by the activation of MCP-1, and the activation of MCP-1 aggravates ECM accumulation by the production of TGF- $\beta 1 .{ }^{28-30}$ The present study also found that NF- $\mathrm{KB}$ and MCP-1 expression were markedly increased in diabetic kidneys and dramatically reduced by treatment with thalidomide. These findings suggest that thalidomide may reduce renal inflammation in DN by negatively regulating the NF- $\mathrm{\kappa B}$ signaling pathway.

Renal fibrosis is one of the main characteristics of DN. TGF- $\beta 1$ is a pivotal mediator in the pathogenesis of DN. ${ }^{31,32}$ Smad proteins are central components of intracellular TGF- $\beta 1$ signaling pathways in the progression of glomerulosclerosis. ${ }^{33} \mathrm{Smad} 3$ is one of the critical downstream mediators in renal fibrosis, and has been shown to be related to the upregulation of fibronectin and collagen IV in DN. ${ }^{34,35}$ Therefore, the regulation of Smads and TGF- $\beta 1$ expression is an effective therapeutic strategy for DN. ${ }^{35,36}$ In our study, STZ-induced diabetic rats exhibited the high expression of TGF- $\beta 1$, T $\beta$ RI, T $\beta$ RII, Smad3, collagen IV, and fibronectin. After thalidomide treatment, diabetic rats showed significant reductions in TGF- $\beta 1$, T $\beta$ RI, T $\beta$ RII, Smad3, fibronectin, and collagen IV, accompanied by less renal injury compared with that in DC rats. These findings are consistent with previous studies in hypertensive nephropathy and obstructive nephropathy. ${ }^{27,37}$ Thus, the protective effect of thalidomide in DN may be associated with inhibition of the TGF- $\beta 1 / \mathrm{Smad} 3$ signaling pathway.

AMPK is an enzyme that takes part in the cellular response to energetic changes. ${ }^{38}$ Prior reports have indicated that the activity of AMPK is reduced in diabetic kidney through multiple pathways. ${ }^{39,40}$ Recent studies have demonstrated that the reduction of $\mathrm{p}$-AMPK promotes the synthesis of abnormal ECM protein and kidney hypertrophy by upregulating mammalian target of rapamycin activity and the TGF- $\beta 1 /$ Smad4 signaling pathway. ${ }^{41,42}$ The activation of AMPK also plays an important role in reducing TGF- $\beta 1$ production in type 1 diabetic renal injury. ${ }^{43}$ Furthermore, previous studies have shown that NF- $\mathrm{KB}$ activation can be inhibited by the activation of AMPK. ${ }^{44,45}$ Therefore, AMPK may serve as a target for the treatment of DN. Consistent with previous

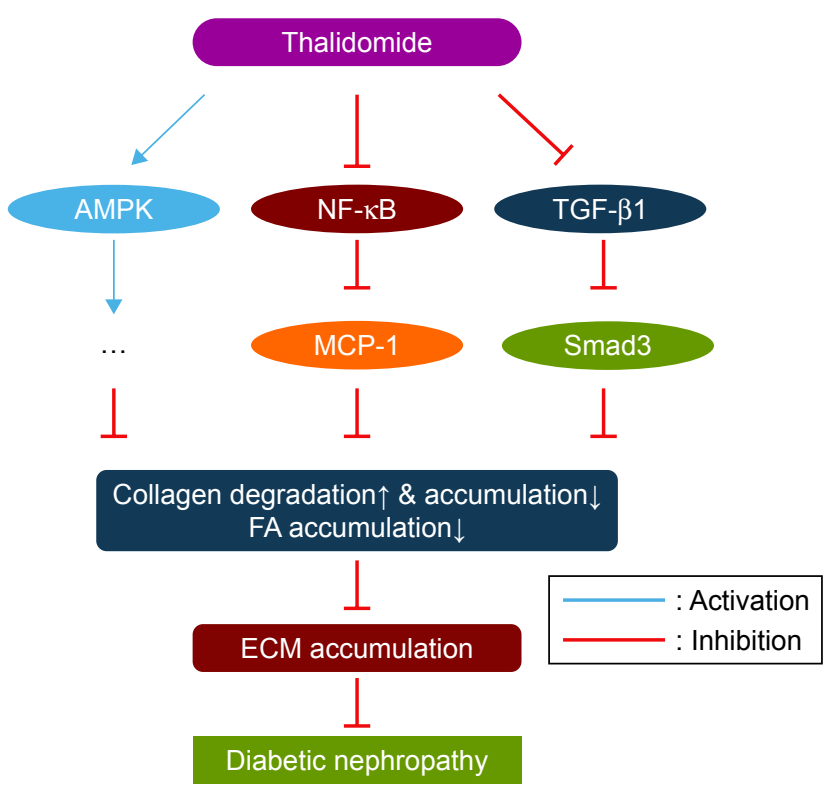

Figure 7 Schematic representation of the proposed mechanism by which thalidomide reduces ECM accumulation through the activation of AMPK $\alpha$ and suppression of NF-KB/MCP-I and TGF- $\beta$ I/Smad3 signaling pathways.

Abbreviations: AMPK $\alpha$, AMP-activated protein kinase $\alpha$; ECM, extracellular matrix; MCP-I, monocyte chemoattractant protein-I; NF- $\kappa B$, nuclear factor- $\kappa B$; TGF- $\beta$ I, transforming growth factor $\beta$ I. 
studies, p-AMPK $\alpha$ expression was markedly reduced and renal inflammation and fibrosis were activated in diabetic rats in the present study. However, after thalidomide treatment, p-AMPK $\alpha$ expression was significantly increased and renal inflammation and fibrosis were markedly reduced, independent of serum glucose levels. Further studies are needed to investigate the mechanism of thalidomide on the AMPK signaling pathway.

In summary, the present study provided the first in vivo evidence that thalidomide reduces renal inflammation and fibrosis through the activation of AMPK $\alpha$, and the inhibition of NF- $\kappa \mathrm{B} / \mathrm{MCP}-1$ and TGF- $\beta 1 / \mathrm{Smad} 3$ signaling in the kidneys of diabetic rats (Figure 7). These results suggest that activation of AMPK $\alpha$ by thalidomide may be a promising target for reducing diabetic renal damage. However, before further clinical investigations, more studies on the molecular mechanism are needed to clarify how exactly thalidomide activates AMPK $\alpha$ in DN.

\section{Disclosure}

The authors report no conflicts of interest in this work.

\section{References}

1. International Diabetes Federation. IDF Diabetes Atlas. 7th ed. Brussels, Belgium: International Diabetes Federation; 2015. Available from: https://idf.org/

2. Lim A. Diabetic nephropathy-complications and treatment. Int J Nephrol Renovasc Dis. 2014;7:361-381.

3. Molitch ME, Adler AI, Flyvbjerg A, et al. Diabetic kidney disease: a clinical update from kidney disease: improving global outcomes. Kidney Int. 2015;87(1):20-30.

4. Adler AI, Stevens RJ, Manley SE, Bilous RW, Cull CA, Holman RR. Development and progression of nephropathy in type 2 diabetes: the United Kingdom Prospective Diabetes Study (UKPDS 64). Kidney Int. 2003;63(1):225-232.

5. Kolset SO, Reinholt FP, Jenssen T. Diabetic nephropathy and extracellular matrix. J Histochem Cytochem. 2012;60(12):976-986.

6. Mason RM, Wahab NA. Extracellular matrix metabolism in diabetic nephropathy. J Am Soc Nephrol. 2003;14(5):1358-1373.

7. Parving HH, Brenner BM, McMurray JJ, et al. Cardiorenal end points in a trial of aliskiren for type 2 diabetes. $N$ Engl J Med. 2012; 367(23):2204-2213.

8. Cooper ME. Diabetes: treating diabetic nephropathy-still an unresolved issue. Nat Rev Endocrinol. 2012;8(9):515-516.

9. Wada J, Makino H. Inflammation and the pathogenesis of diabetic nephropathy. Clin Sci (Lond). 2013;124(3):139-152.

10. Garcia-Garcia PM, Getino-Melian MA, Dominguez-Pimentel V, Navarro-Gonzalez JF. Inflammation in diabetic kidney disease. World $J$ Diabetes. 2014;5(4):431-443.

11. Arai H, Furusu A, Nishino T, et al. Thalidomide prevents the progression of peritoneal fibrosis in mice. Acta Histochem Cytochem. 2011; 44(2):51-60.

12. Choe JY, Jung HJ, Park KY, et al. Anti-fibrotic effect of thalidomide through inhibiting TGF-beta-induced ERK1/2 pathways in bleomycin induced lung fibrosis in mice. Inflamm Res. 2010;59(3):177-188.

13. Mondello S, Mazzon E, Di Paola R, et al. Thalidomide suppresses sclerosing encapsulating peritonitis in a rat experimental model. Shock. 2009;32(3):332-339.
14. Tabata C, Tabata R, Kadokawa Y, et al. Thalidomide prevents bleomycin-induced pulmonary fibrosis in mice. J Immunol. 2007;179(1): 708-714.

15. Yndestad A, Vinge LE, Bjørnerheim R, et al. Thalidomide attenuates the development of fibrosis during post-infarction myocardial remodelling in rats. Eur J Heart Fail. 2006;8(8):790-796.

16. Santana AC, Degaspari S, Catanozi S, et al. Thalidomide suppresses inflammation in adenine-induced CKD with uraemia in mice. Nephrol Dial Transplant. 2013;28(5):1140-1149.

17. Zhao J, Wang H, Song T, Yang Y, Gu K, Ma P. Thalidomide promotes morphine efficacy and prevents morphine-induced tolerance in rats with diabetic neuropathy. Neurochem Res. 2016;41(12):3171-3180.

18. Behl T, Kaur I, Goel H, Kotwani A. Significance of the antiangiogenic mechanisms of thalidomide in the therapy of diabetic retinopathy. Vascul Pharmacol. 2017;92:6-15.

19. Kim DH, Kim YJ, Chang SA, et al. The protective effect of thalidomide on left ventricular function in a rat model of diabetic cardiomyopathy. Eur J Heart Fail. 2010;12(10):1051-1060.

20. Bosco AA, Lerario AC, Santos RF, Wajchenberg BL. Effect of thalidomide and rosiglitazone on the prevention of diabetic retinopathy in streptozotocin-induced diabetic rats. Diabetologia. 2003;46(12): $1669-1675$.

21. Kitada M, Kume S, Imaizumi N, Koya D. Resveratrol improves oxidative stress and protects against diabetic nephropathy through normalization of Mn-SOD dysfunction in AMPK/SIRT1- independent pathway. Diabetes. 2011;60(2):634-643.

22. Zhao T, Sun S, Zhang H, et al. Therapeutic effects of tangshen formula on diabetic nephropathy in rats. PLoS One. 2016;11(1): e0147693.

23. Like AA, Rossini AA. Streptozotocin-induced pancreatic insulitis: new model of diabetes mellitus. Science. 1976;193(4251):415-417.

24. Wang C, Pan Y, Zhang QY, Wang FM, Kong LD. Quercetin and allopurinol ameliorate kidney injury in STZ-treated rats with regulation of renal NLRP3 inflammasome activation and lipid accumulation. PLoS One. 2012;7(6):e38285.

25. Kraynak AR, Storer RD, Jensen RD, et al. Extent and persistence of streptozotocin-induced DNA damage and cell proliferation in rat kidney as determined by in vivo alkaline elution and BrdUrd labeling assays. Toxicol Appl Pharmacol. 1995;135(2):279-286.

26. Ortega A, Fernández A, Arenas MI, et al. Outcome of acute renal injury in diabetic mice with experimental endotoxemia: role of hypoxiainducible factor-1 $\alpha$. J Diabetes Res. 2013;2013:254529.

27. Williams MD, Nadler JL. Inflammatory mechanisms of diabetic complications. Curr Diab Rep. 2007;7(3):242-248.

28. Tesch GH. MCP-1/CCL2: a new diagnostic marker and therapeutic target for progressive renal injury in diabetic nephropathy. Am J Physiol Renal Physiol. 2008;294(4):697-701.

29. Morii T, Fujita H, Narita T, et al. Association of monocyte chemoattractant protein-1 with renal tubular damage in diabetic nephropathy. J Diabetes Complications. 2003;17(1):11-15.

30. Cheng J, Diaz Encarnacion MM, Warner GM, Gray CE, Nath KA, Grande JP. TGF-beta1 stimulates monocyte chemoattractant protein-1 expression in mesangial cells through a phosphodiesterase isoenzyme 4-dependent process. Am J Physiol Cell Physiol. 2005;289(4):959-970.

31. Ahn JY, Kim MH, Lim MJ, et al. The inhibitory effect of ginsan on TGF- $\beta$ mediated fibrotic process. J Cell Physiol. 2011;226(5): 1241-1247.

32. Luo F, Zhuang Y, Sides MD, Sanchez CG, Shan B, White ES. Arsenic trioxide inhibits transforming growth factor- $\beta 1$-induced fibroblast to myofibroblast differentiation in vitro and bleomycin induced lung fibrosis in vivo. Respir Res. 2014;15:51.

33. Wang W, Koka V, Lan HY. Transforming growth factor-beta and smad signalling in kidney diseases. Nephrology. 2005;10(1):48-56.

34. Wang A, Ziyadeh FN, Lee EY, et al. Interference with TGF-beta signaling by Smad3-knockout in mice limits diabetic glomerulosclerosis without affecting albuminuria. Am J Physiol Renal Physiol. 2007;293(5): F1657-F1665. 
35. Fujimoto M, Maezawa Y, Yokote K, et al. Mice lacking Smad3 are protected against streptozotocin-induced diabetic glomerulopathy. Biochem Biophys Res Commun. 2003;305(4):1002-1007.

36. Schiffer M, Schiffer LE, Gupta A, et al. Inhibitory smads and TGFbeta signaling in glomerular cells. J Am Soc Nephrol. 2002;13(11): 2657-2666.

37. Li JH, Huang XR, Zhu HJ, et al. Advanced glycation end products activate Smad signaling via TGF-beta-dependent and independent mechanisms: implications for diabetic renal and vascular disease. FASEB J. 2004;18(1):176-178.

38. Kemp BE, Stapleton D, Campbell DJ, et al. AMP-activated protein kinase, super metabolic regulator. Biochem Soc Trans. 2003;31(Pt1): $162-168$.

39. Decleves AE, Mathew AV, Cunard R, Sharma K. AMPK mediates the initiation of kidney disease induced by a high-fat diet. J Am Soc Nephrol. 2011;22(10):1846-1855.

40. Hallows KR, Mount PF, Pastor-Soler NM, Power DA. Role of the energy sensor AMP-activated protein kinase in renal physiology and disease. Am J Physiol Renal Physiol. 2010;298(5):1067-1077.
41. Lee MJ, Feliers D, Mariappan MM, et al. A role for AMP-activated protein kinase in diabetes-induced renal hypertrophy. Am J Physiol Renal Physiol. 2007;292(2):617-627.

42. Zhao J, Miyamoto S, You YH, Sharma K. AMP-activated protein kinase (AMPK) activation inhibits nuclear translocation of Smad4 in mesangial cells and diabetic kidneys. Am J Physiol Renal Physiol. 2015; 308(10):F1167-F1177.

43. Dugan LL, You YH, Ali SS, et al. AMPK dysregulation promotes diabetes-related reduction of superoxide and mitochondrial function. J Clin Invest. 2013;123(11):4888-4899.

44. Salminen A, Hyttinen JM, Kaarniranta K. AMP-activated protein kinase inhibits NF-kappaB signaling and inflammation: impact on health span and lifespan. J Mol Med. 2011;89(7):667-676.

45. Wang S, Zhang M, Liang B, et al. AMPKalpha2 deletion causes aberrant expression and activation of $\mathrm{NAD}(\mathrm{P}) \mathrm{H}$ oxidase and consequent endothelial dysfunction in vivo: role of $26 \mathrm{~S}$ proteasomes. Circ Res. 2010; 106(6):1117-1128.

\section{Publish your work in this journal}

Drug Design, Development and Therapy is an international, peerreviewed open-access journal that spans the spectrum of drug design and development through to clinical applications. Clinical outcomes, patient safety, and programs for the development and effective, safe, and sustained use of medicines are the features of the journal, which has also been accepted for indexing on PubMed Central. The manuscript management system is completely online and includes a very quick and fair peer-review system, which is all easy to use. Visit http://www.dovepress.com/testimonials.php to read real quotes from published authors.

Submit your manuscript here: http://www.dovepress.com/drug-design-development-and-therapy-journal 